\title{
ON A RESULT OF SINGH
}

\section{HONG-XUN YI}

In this paper relations between $T(r, f)$ and $T\left(r, f^{(k)}\right)$ are established for a class of meromorphic functions $f(z)$, where $T(r, f)$ and $T\left(r, f^{(k)}\right)$ are the Nevanlinna characteristic functions $f(z)$ and $f^{(k)}(z)$ respectively. An example is provided to show that a result of Singh is not true. The conclusions obtained here correct and generalise the result of Singh.

We denote by $C$ the set of all finite complex numbers and by $\bar{C}$ the extended complex plane consisting of all (finite) complex numbers and $\infty$. Let $f(z)$ be a transcendental meromorphic function in the complex plane. We use with their usual definitions the Nevanlinna functions $T(r, f), N(r, f)$, et cetera (see [1]). If $f(z)-a$ has a finite number of simple zeros, we say that $a$ is an exceptional value Picard (e.v.P.) for simple zeros of $f(z)$. If $f(z)$ has a finite number of simple poles, we say that $\infty$ is e.v.P. for simple zeros of $f(z)$. In [3] Singh obtained the following result:

Let $f(z)$ be a transcendental meromorphic function of finite order with four (finite or infinite) distinct e.v.P. for simple zeros. Then

$$
\lim _{r \rightarrow \infty} \frac{T\left(r, f^{\prime}\right)}{T(r, f)}=\frac{3}{2}
$$

Let $f(z)=s n(z)$, where $s n(z)$ is the Jacobian elliptic function (see [2]). We know that $f(z)$ is a transcendental meromorphic function of finite order and that $\left(f^{\prime}\right)^{2}=$ $\left(1-f^{2}\right)\left(1-t^{2} f^{2}\right)$, where $t(\neq 0,1,-1)$ is a constant. It is easy to see that $1,-1,1 / t$ and $-1 / t$ are four distinct e.v.P. for simple zeros of $f(z)$ and that

$$
\lim _{r \rightarrow \infty} \frac{T\left(r, f^{\prime}\right)}{T(r, f)}=2
$$

This shows that the above result of Singh is wrong.

In this paper we obtain the following theorem which is a correction of the result of Singh.

Received 22 June 1989

Copyright Clearance Centre, Inc. Serial-fee code: 0004-9729/90 SA2.00+0.00. 
THEOREM 1. Let $f(z)$ be a transcendental meromorphic function of finite order with four distinct e.v.P. for simple zeros.

(i) If $\infty$ is an e.v.P. for simple zeros of $f(z)$, then

$$
\lim _{r \rightarrow \infty} \frac{T\left(r, f^{\prime}\right)}{T(r, f)}=\frac{3}{2}
$$

(ii) If $\infty$ is not an e.v.P. for simple zeros of $f(z)$, then

$$
\lim _{r \rightarrow \infty} \frac{T\left(r, f^{\prime}\right)}{T(r, f)}=2 .
$$

Instead of Theorem 1, we prove the more general theorem:

THEOREM 2. Let $f(z)$ be a transcendental meromorphic function of finite order with four distinct e.v.P. for simple zeros and $k$ be a positive integer.

(i) If $\infty$ is an e.v.P. for simple zeros of $f(z)$, then

$$
\lim _{r \rightarrow \infty} \frac{T\left(r, f^{(k)}\right)}{T(r, f)}=\frac{1}{2} k+1 ;
$$

(ii) If $\infty$ is not an e.v.P. for simple zeros of $f(z)$, then

$$
\lim _{r \rightarrow \infty} \frac{T\left(r, f^{(k)}\right)}{T(r, f)}=k+1
$$

In order to state our third theorem, we introduce the following notation.

Let $f(z)$ be a meromorphic function and $a \in \bar{C}$. We denote by $n_{1}(r, a, f)$ the number of simple zeros of $f(z)-a$ in $|z| \leqslant r . \quad N_{1}(r, a, f)$ is defined in terms of $n_{1}(r, a, f)$ in the usual way. Further we define

$$
\delta_{1}(a, f)=1-\limsup _{r \rightarrow \infty} \frac{N_{1}(r, a, f)}{T(r, f)} .
$$

Yang [4] proved that there exists at most a denumerable number of complex numbers $a$ for which $\delta_{1}(a, f)>0$ and

$$
\sum_{a \in \bar{C}} \delta_{1}(a, f) \leqslant 4
$$

THEOREM 3. Let $f(z)$ be a transcendental meromorphic function of finite order and $k$ be a positive integer. If

$$
\sum_{a \in \bar{C}} \delta_{1}(a, f)=4
$$


then

$$
\lim _{r \rightarrow \infty} \frac{T\left(r, f^{(k)}\right)}{T(r, f)}=k+1-\frac{1}{2} k \delta_{1}(\infty, f) .
$$

Obviously, if $a$ is an e.v.P. for simple zeros of $f(z)$, then $\delta_{1}(a, f)=1$.

Thus Theorem 3 includes Theorem 2 as a very special case.

Proof of Theorem 3: Let $\left\{a_{i}\right\}_{i=1}^{\infty}$ be an infinite sequence of distinct elements of $C$ which includes every $a \in C$ satisfying $\delta_{1}(a, f)>0$. By the second fundamental theorem and noting that $f(z)$ is a transcendental meromorphic function of finite order, we have

$$
(q-1) T(r, f)<\sum_{i=1}^{q} \bar{N}\left(r, a_{i}, f\right)+\bar{N}(r, f)+O(\log r),
$$

where $q$ is any positive integer. Again

$$
\begin{aligned}
\bar{N}\left(r, a_{i}, f\right) & \leqslant \frac{1}{2} N_{1}\left(r, a_{i}, f\right)+\frac{1}{2} N\left(r, a_{i}, f\right) \\
& \leqslant \frac{1}{2} N_{1}\left(r, a_{i}, f\right)+\frac{1}{2} T(r, f)+O(1) .
\end{aligned}
$$

From (2) and (3) we obtain

$$
(q-1) T(r, f)<\frac{1}{2} \sum_{i=1}^{q} N_{1}\left(r, a_{i}, f\right)+\frac{1}{2} q T(r, f)+\bar{N}(r, f)+O(\log r) .
$$

Thus

$$
\liminf _{r \rightarrow \infty} \frac{\bar{N}(r, f)}{T(r, f)} \geqslant \frac{1}{2} \sum_{i=1}^{q} \delta_{1}\left(a_{i}, f\right)-1
$$

Since (5) holds for all $q \geqslant 1$, letting $q \rightarrow \infty$, we get

$$
\begin{aligned}
\liminf _{r \rightarrow \infty} \frac{\bar{N}(r, f)}{T(r, f)} & \geqslant \frac{1}{2} \sum_{a \in C} \delta_{1}(a, f)-1 \\
& =1-\frac{1}{2} \delta_{1}(\infty, f)
\end{aligned}
$$

using (1).

On the other hand,

$$
\bar{N}(r, f) \leqslant \frac{1}{2} N_{1}(r, f)+\frac{1}{2} N(r, f)
$$


and hence

$$
\bar{N}(r, f) \leqslant \frac{1}{2} N_{1}(r, f)+\frac{1}{2} T(r, f) .
$$

Thus

$$
\underset{r \rightarrow \infty}{\limsup } \frac{\bar{N}(r, f)}{T(r, f)} \leqslant 1-\frac{1}{2} \delta_{1}(\infty, f) .
$$

From (6) and (9) we obtain

$$
\lim _{r \rightarrow \infty} \frac{\bar{N}(r, f)}{T(r, f)}=1-\frac{1}{2} \delta_{1}(\infty, f)
$$

By (7) and (10) we have

$$
\lim _{r \rightarrow \infty} \frac{N(r, f)}{T(r, f)}=1
$$

using $N(r, f) \leqslant T(r, f)$.

Since

and

$$
\begin{aligned}
& N\left(r, f^{(k)}\right)=N(r, f)+k \bar{N}(r, f) \\
& m\left(r, f^{(k)}\right)<m(r, f)+O(\log r),
\end{aligned}
$$

thus we have

$$
N(r, f)+k \bar{N}(r, f)<T\left(r, f^{(k)}\right)<T(r, f)+k \bar{N}(r, f)+O(\log r) .
$$

From (10), (11) and (12), we get

$$
\lim _{r \rightarrow \infty} \frac{T\left(r, f^{(k)}\right)}{T(r, f)}=k+1-\frac{1}{2} k \delta_{1}(\infty, f)
$$

This completes the proof of Theorem 3.

\section{REFERENCES}

[1] W.K. Hayman, Meromorphic Functions (Oxford Univ. Press, 1964).

[2] E.D. Rainville, Special Functions (Macmillan Company, New York, 1960).

[3] A.P. Singh, 'A note on exceptional value Picard for simple zeros', Progr. Math. 15 (1981), 9-11.

[4] L. Yang, Value distribution theory and new research on it (Science Press, Beijing, 1982).

Department of Mathematics

Shandong University

Jinan

Shandong 250100

China 